\title{
On the Analytical Treatment of Singular Impedance Integrals in Electromagnetics
}

\author{
I. Bogaert*
}

\begin{abstract}
A method is presented for reducing the number of subsequent integrations in impedance integrals containing the dynamic Green's function. The method works whenever a point can be found that is coplanar (or collinear) to both the basis and test support. This is always the case for singular impedance integrals. As an example, explicit formulas are given for the self-patch impedance integrals for the electric field integral equation.
\end{abstract}

\section{Introduction}

The accurate numerical evaluation of impedance integrals for boundary or volume integral equations is of great importance in computational electromagnetics. Indeed, errors on the impedance integrals influence the final solution accuracy, either directly or indirectly, as is for example the case at low frequencies in the mixed MFIE $[1,2]$.

However, achieving a high accuracy is not a goal that is easily accomplished. The main reason for this of course being the presence of a singular Green's function. Standard quadrature rules can only handle smooth functions or functions with very specific singularities. Therefore, impedance integrals for touching or overlapping basis and test supports cannot be accurately computed using such standard rules. Unfortunately, these so-called singular integrals are also the most important to evaluate accurately, since they constitute the strongest interactions. To solve this problem, much attention has gone to the development of numerical and semianalytical techniques to compute these impedance integrals. Among the most widely used techniques are the singularity subtraction [3-6] and singularity cancelation [7-10] techniques.

Another research avenue has been to construct analytical expressions for impedance integrals. These have usually been limited to static Green's functions or the more general power-law Green's functions. For example, analytical expressions for the complete self-patch integral with Green's functions $r^{\nu}, \nu \in[-1,4]$ (with $r$ the distance between source and observer locations) can be found in $[11,12]$. Recently, the case for general triangles was solved for basis functions proportional to the position vector [13] and constant test functions (or vice

\footnotetext{
*Department of Information Technology, Ghent University, Sint-Pietersnieuwstraat 41, 9000 Ghent, Belgium, email:Ignace.Bogaert@intec.UGent . be
}

versa).

In this contribution, a technique is proposed that allows singular impedance integrals with the $d y$ namic Green's function to be reduced to double or single integrals in an analytically exact way. The remaining double or single integrals are nonsingular and can therefore be numerically computed to high accuracy with acceptable computational resources. The method can be applied if both the basis and test functions are polynomial functions with polygonal support.

\section{Brief Derivation for a Triangle-Line Inte- gral}

In [14], the impedance integral between a constant test function on a line support $\Gamma_{t}=\left\{\boldsymbol{t}_{1}, \boldsymbol{t}_{2}\right\}$ and a constant basis function on a triangular support $\Gamma_{b}=\left\{\boldsymbol{b}_{1}, \boldsymbol{b}_{2}, \boldsymbol{b}_{3}\right\}$ was considered:

$$
Z_{\nu}=\int_{0}^{1} \int_{0}^{1} \int_{0}^{1-u_{b}}\left\|\boldsymbol{r}\left(u_{b}, v_{b}\right)-\boldsymbol{r}^{\prime}\left(u_{t}\right)\right\|^{\nu} \mathrm{d} v_{b} \mathrm{~d} u_{b} \mathrm{~d} u_{t}
$$

with

$$
\begin{aligned}
\boldsymbol{r}\left(u_{b}, v_{b}\right) & =u_{b} \boldsymbol{b}_{1}+v_{b} \boldsymbol{b}_{2}+\left(1-u_{b}-v_{b}\right) \boldsymbol{b}_{3}, \\
\boldsymbol{r}^{\prime}\left(u_{t}\right) & =u_{t} \boldsymbol{t}_{1}+\left(1-u_{t}\right) \boldsymbol{b}_{2} .
\end{aligned}
$$

A way to convert this triple integral to five double integrals was derived, using the fact that the Green's function had a power-law behavior, i.e. it was essentially scale-invariant. The result reads

$$
\begin{aligned}
& (\nu+3) Z_{\nu}= \\
& u_{t 0} \int_{0}^{1} \int_{0}^{1-u_{b}}\left\|\boldsymbol{r}(0)-\boldsymbol{r}^{\prime}\left(u_{b}, v_{b}\right)\right\|^{\nu} \mathrm{d} v_{b} \mathrm{~d} u_{b} \\
& +v_{t 0} \int_{0}^{1} \int_{0}^{1-u_{b}}\left\|\boldsymbol{r}(1)-\boldsymbol{r}^{\prime}\left(u_{b}, v_{b}\right)\right\|^{\nu} \mathrm{d} v_{b} \mathrm{~d} u_{b} \\
& +u_{b 0} \int_{0}^{1} \int_{0}^{1}\left\|\boldsymbol{r}\left(u_{t}\right)-\boldsymbol{r}^{\prime}\left(0, v_{b}\right)\right\|^{\nu} \mathrm{d} v_{b} \mathrm{~d} u_{t} \\
& +v_{b 0} \int_{0}^{1} \int_{0}^{1}\left\|\boldsymbol{r}\left(u_{t}\right)-\boldsymbol{r}^{\prime}\left(u_{b}, 0\right)\right\|^{\nu} \mathrm{d} u_{b} \mathrm{~d} u_{t} \\
& +w_{b 0} \int_{0}^{1} \int_{0}^{1}\left\|\boldsymbol{r}\left(u_{t}\right)-\boldsymbol{r}^{\prime}\left(u_{b}, 1-u_{b}\right)\right\|^{\nu} \mathrm{d} u_{b} \mathrm{~d} u_{t},
\end{aligned}
$$


with the coefficients defined by

$$
\begin{aligned}
\boldsymbol{r}\left(u_{b 0}, v_{b 0}\right) & =\boldsymbol{r}^{\prime}\left(u_{t 0}\right), \\
w_{b 0} & =1-u_{b 0}-v_{b 0}, \\
v_{t 0} & =1-u_{t 0} .
\end{aligned}
$$

In this contribution, the reduction (4) will be extended to the dynamic Green's function

$$
G(r)=\frac{e^{-i k r}}{4 \pi r}=-\frac{i k}{4 \pi} \sum_{\nu=-1}^{\infty} \frac{(-i k r)^{\nu}}{(\nu+1) !} .
$$

To keep the notation simple and the focus in line with the topic of this paper, the triangle and line are assumed to be touching in one or more points:

$$
\boldsymbol{t}_{2}=\boldsymbol{b}_{3} .
$$

In this case, (4) simplifies to

$$
\begin{array}{r}
(\nu+3) Z_{\nu}=\int_{0}^{1} \int_{0}^{1-u_{b}}\left\|\boldsymbol{r}(1)-\boldsymbol{r}^{\prime}\left(u_{b}, v_{b}\right)\right\|^{\nu} \mathrm{d} v_{b} \mathrm{~d} u_{b} \\
+\int_{0}^{1} \int_{0}^{1}\left\|\boldsymbol{r}\left(u_{t}\right)-\boldsymbol{r}^{\prime}\left(u_{b}, 1-u_{b}\right)\right\|^{\nu} \mathrm{d} u_{b} \mathrm{~d} u_{t}
\end{array}
$$

Now, applying the summation operator

$$
S[\boldsymbol{g}]=-\frac{i k}{4 \pi} \sum_{\nu=-1}^{\infty} \frac{(-i k)^{\nu}}{(\nu+1) !(\nu+3)} \boldsymbol{g}_{\nu}
$$

to the left hand side of (10) yields

$$
\begin{aligned}
& S\left[(\nu+3) Z_{\nu}\right]=-\frac{i k}{4 \pi} \sum_{\nu=-1}^{\infty} \frac{(-i k)^{\nu}}{(\nu+1) !} Z_{\nu}, \\
& =\int_{0}^{1} \int_{0}^{1} \int_{0}^{1-u_{b}} G\left(\left\|\boldsymbol{r}\left(u_{b}, v_{b}\right)-\boldsymbol{r}^{\prime}\left(u_{t}\right)\right\|\right) \mathrm{d} v_{b} \mathrm{~d} u_{b} \mathrm{~d} u_{t} .
\end{aligned}
$$

Interchanging the summation and the integration is allowed because of Fubini's theorem. The result (12) is an impedance integral with the dynamic Green's function. To show that this impedance integral can also be written as the sum of two double integrals, we have to apply the summation operator (11) to the right hand side of (10). This results in

$$
\begin{aligned}
S\left[\int_{0}^{1}\right. & \left.\int_{0}^{1-u_{b}}\left\|\boldsymbol{r}(1)-\boldsymbol{r}^{\prime}\left(u_{b}, v_{b}\right)\right\|^{\nu} \mathrm{d} v_{b} \mathrm{~d} u_{b}\right]= \\
& \int_{0}^{1} \int_{0}^{1-u_{b}} G_{3}\left(\left\|\boldsymbol{r}(1)-\boldsymbol{r}^{\prime}\left(u_{b}, v_{b}\right)\right\|\right) \mathrm{d} v_{b} \mathrm{~d} u_{b},
\end{aligned}
$$

and

$$
\begin{aligned}
S\left[\int_{0}^{1}\right. & \left.\int_{0}^{1}\left\|\boldsymbol{r}\left(u_{t}\right)-\boldsymbol{r}^{\prime}\left(u_{b}, 1-u_{b}\right)\right\|^{\nu} \mathrm{d} u_{b} \mathrm{~d} u_{t}\right]= \\
& \int_{0}^{1} \int_{0}^{1} G_{3}\left(\left\|\boldsymbol{r}\left(u_{t}\right)-\boldsymbol{r}^{\prime}\left(u_{b}, 1-u_{b}\right)\right\|\right) \mathrm{d} u_{b} \mathrm{~d} u_{t} .
\end{aligned}
$$

Here, the function $G_{3}$ is given by

$$
G_{3}(r)=S\left[r^{\nu}\right]=-\frac{i k}{4 \pi} \frac{1+(w-1) e^{w}}{w^{3}},
$$

with $w=-i k r$.

Summarizing, it has been shown that

$$
\begin{aligned}
& \int_{\Gamma_{t}} \int_{\Gamma_{b}} G\left(\left\|\boldsymbol{r}-\boldsymbol{r}^{\prime}\right\|\right) \mathrm{d} \boldsymbol{r}^{\prime} \mathrm{d} \boldsymbol{r}= \\
& \quad \int_{0}^{1} \int_{0}^{1-u_{b}} G_{3}\left(\left\|\boldsymbol{r}(1)-\boldsymbol{r}^{\prime}\left(u_{b}, v_{b}\right)\right\|\right) \mathrm{d} v_{b} \mathrm{~d} u_{b} \\
& \quad+\int_{0}^{1} \int_{0}^{1} G_{3}\left(\left\|\boldsymbol{r}\left(u_{t}\right)-\boldsymbol{r}^{\prime}\left(u_{b}, 1-u_{b}\right)\right\|\right) \mathrm{d} u_{b} \mathrm{~d} u_{t} .
\end{aligned}
$$

This is a reduction of the number of integrations from three to two, for an impedance integral containing the dynamic Green's function. The fact that this reduction is possible is because the dimension reduction method presented in [14] leaves power-law Green's functions invariant. This allows the summation operator $S$ to be applied to a function that has a similar structure as the original Green's function, which enables the analytical evaluation of the sum in this case.

It is worthwhile to point out that this summation can also be applied to the more general (4), yielding identical results. This is of significant practical importance for the evaluation of impedance integrals for the razor-blade tested MFIE $[15,16]$. Indeed, up to a change in the Green's function, the impedance integrals have exactly the same structure as (4).

\section{Self-Patch Formulas}

The technique described in the above can be used to convert many pertinent singular impedance integrals to lower-dimensional non-singular ones. Here, formulas will be given for the case of the self-patch for the electric field integral equation on a triangular mesh. The basis and test function are assumed to be linear, which is true in for example an RWG discretization. Arguably, this case has practical importance for the discretization of boundary integral equations.

First, some notation will be introduced. Let the triangular support $\Gamma$ have vertices $\boldsymbol{r}_{1}, \boldsymbol{r}_{2}$ and $\boldsymbol{r}_{3}$. 
Because of their linearity, the basis and testing functions can be written as linear combinations of the barycentric coordinates. This means that the nine self-patch integrals with a barycentric coordinate as basis and test function provide a sufficient set of integrals to compute all possible self-patches for the actual basis and test functions. Due to symmetry reasons, only two cases need to be treated:

$$
\begin{aligned}
& I_{u u}=\int_{0}^{1} \int_{0}^{1-u} \int_{0}^{1} \int_{0}^{1-u^{\prime}} u u^{\prime} G(r) \mathrm{d} u \mathrm{~d} v \mathrm{~d} u^{\prime} \mathrm{d} v^{\prime}, \\
& I_{u v}=\int_{0}^{1} \int_{0}^{1-u} \int_{0}^{1} \int_{0}^{1-u^{\prime}} u v^{\prime} G(r) \mathrm{d} u \mathrm{~d} v \mathrm{~d} u^{\prime} \mathrm{d} v^{\prime},
\end{aligned}
$$

with $r=\left\|\left(u-u^{\prime}\right)\left(\boldsymbol{r}_{1}-\boldsymbol{r}_{3}\right)+\left(v-v^{\prime}\right)\left(\boldsymbol{r}_{2}-\boldsymbol{r}_{3}\right)\right\|$. Formulas for the other seven integrals can be obtained by cyclic permutation of the vertices or by invoking the invariance of the Green's function under inversion.

Now the formulas for integrals (17) and (18) will be given. First define

$$
I_{\boldsymbol{r}_{1}, \boldsymbol{r}_{2}, \boldsymbol{r}_{3}}^{F}(h(t))=\int_{0}^{1} h(t) F\left(\left\|t\left(\boldsymbol{r}_{1}-\boldsymbol{r}_{2}\right)+\boldsymbol{r}_{2}-\boldsymbol{r}_{3}\right\|\right) \mathrm{d} t .
$$

Using this notation, the following holds

$$
\begin{aligned}
I_{u u}= & 2\left[I_{\boldsymbol{r}_{3}, \boldsymbol{r}_{1}, \boldsymbol{r}_{2}}^{G_{c}}(1-t)+I_{\boldsymbol{r}_{1}, \boldsymbol{r}_{2}, \boldsymbol{r}_{3}}^{G_{r_{2}}}(t)+I_{\boldsymbol{r}_{2}, \boldsymbol{r}_{3}, \boldsymbol{r}_{1}}^{G_{b}}(1)\right] \\
& +4\left[I_{\boldsymbol{r}_{3}, \boldsymbol{r}_{1}, \boldsymbol{r}_{2}}^{G_{2}}(1)+I_{\boldsymbol{r}_{1}, \boldsymbol{r}_{2}, \boldsymbol{r}_{3}}^{G_{2}}(1)\right],
\end{aligned}
$$

and

$$
\begin{aligned}
I_{u v} & =I_{\boldsymbol{r}_{2}, \boldsymbol{r}_{3}, \boldsymbol{r}_{1}}^{G_{a}}(t)+I_{\boldsymbol{r}_{3}, \boldsymbol{r}_{1}, \boldsymbol{r}_{2}}^{G_{a}}(1-t) \\
& +I_{\boldsymbol{r}_{3}, \boldsymbol{r}_{1}, \boldsymbol{r}_{2}}^{G_{3}}(1)+I_{\boldsymbol{r}_{1}, \boldsymbol{r}_{2}, \boldsymbol{r}_{3}}^{G_{b}}(1)+I_{\boldsymbol{r}_{2}, \boldsymbol{r}_{3}, \boldsymbol{r}_{1}}^{G_{b}}(1) .
\end{aligned}
$$

Here, the following Green's functions occur:

$$
\begin{aligned}
& G_{a}(r)=\frac{8-5 w+w^{2}}{w^{6}} e^{w}+\frac{w^{3}-48-18 w}{6 w^{6}}, \\
& G_{b}(r)=\frac{w-2}{w^{6}} e^{w}-\frac{w^{4}+2 w^{3}-12 w-24}{12 w^{6}} \\
& G_{c}(r)=\frac{w-4}{w^{6}} e^{w}+\frac{w^{3}+6 w^{2}+18 w+24}{6 w^{6}} \\
& G_{d}(r)=\frac{e^{w}}{w^{6}}-\frac{w^{4}+4 w^{3}+12 w^{2}+24 w+24}{24 w^{6}}
\end{aligned}
$$

again with $w=-i k r$.

Formulas (20) and (21) hold for arbitrary complex wave numbers $k$ and for any triangle. The remaining integrals are one-dimensional and, in addition, do not contain singularities because they always involve interactions between an edge of the triangle and the overlying vertex. In case one of the vertices is very close to the overlying edge (e.g. when a low-quality mesh is used), techniques such as that described in [8] can be used to compute the one-dimensional integrals.

\section{Numerical Results}

Formula (20) was tested on a triangle with vertices

$$
\begin{aligned}
& \boldsymbol{r}_{1}=[\pi, 1, \sqrt{2}], \\
& \boldsymbol{r}_{2}=[0,0,0], \\
& \boldsymbol{r}_{3}=\left[\frac{1}{\pi}, 0, e\right] .
\end{aligned}
$$

Figure 1 shows the behavior of the self-patch (17) for varying wave number $k$. The result is compared to the same integral, computed using the results from [12], where the series is truncated after the term containing $k^{5}$. Of course, these two results are only the same up to a term scaling like $\mathcal{O}\left(k^{6}\right)$, which nicely explains the discrepancy for higher frequencies.

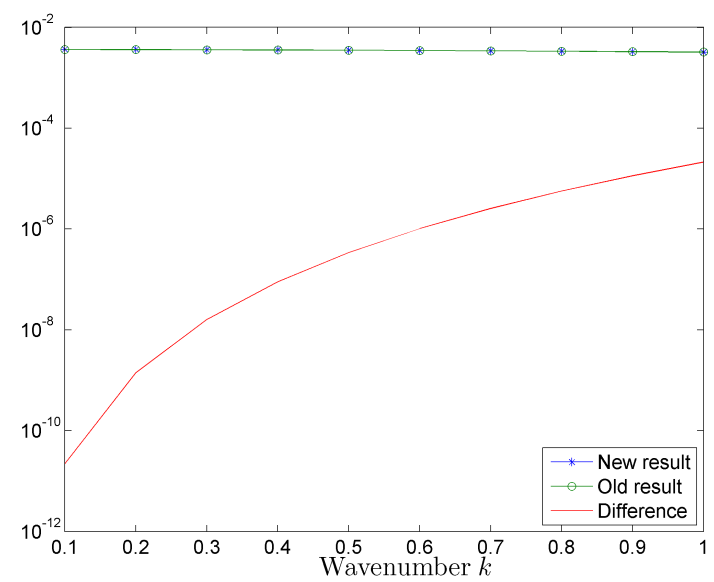

Figure 1: A comparison of the absolute value of integral (17), obtained using formula (20) and the results from [12]. The absolute value of the difference is also given and follows the expected $\mathcal{O}\left(k^{6}\right)$ behavior.

\section{Conclusion}

A method was presented for extending dimensionality reduction of impedance integrals from powerlaw Green's functions to the dynamic Green's function. It was illustrated by reducing a singular triple integral to a sum of two double integrals, and by providing formulas for the self-patch of the electric field integral equation. The remaining integrals in these formulas contain only one-dimensional and 
nonsingular integrals and can be evaluated using standard techniques.

\section{Acknowledgments}

This work was supported by a postdoctoral grant from the Fund for Scientific Research Flanders (FWO-Vlaanderen).

\section{References}

[1] I. Bogaert, K. Cools, F. P. Andriulli, J. Peeters, and D. De Zutter, "Low Frequency Stability of the Mixed Discretization of the MFIE," in Proceedings of the 5th European Conference on Antennas and Propagation, Rome, Italy, 11-15 April 2011.

[2] K. Cools, F. Andriulli, D. De Zutter, and E. Michielssen, "Accurate and Conforming Mixed Discretization of the MFIE," IEEE Antennas and Wireless Propagation Letters, vol. 10, pp. 528-531, 2011.

[3] D. R. Wilton, S. M. Rao, D. H. S. A. W. Glisson, O. M. Al-Bundak, and C. M. Butler, "Potential integrals for uniform and linear source distributions on polygonal and polyhedral domains," IEEE Trans. Antennas Propag., vol. AP-32, no. 3, Mar. 1984.

[4] C. Butler, "Evaluation of potential integral at singularity of exact kernel in thin-wire calculations," Antennas and Propagation, IEEE Transactions on, vol. 23, no. 2, pp. $293-295$, mar 1975.

[5] S. Jarvenpaa, M. Taskinen, and P. Yla-Oijala, "Singularity subtraction technique for highorder polynomial vector basis functions on planar triangles," Antennas and Propagation, IEEE Transactions on, vol. 54, no. 1, pp. $42-$ 49, jan. 2006.

[6] I. Hanninen, M. Taskinen, and J. Sarvas, "Singularity subtraction integral formulae for surface integral equations with rwg, rooftop and hybrid basis functions," Progress In Electromagnetics Research, vol. 63, pp. 243-278, 2006.

[7] M. A. Khayat and D. R. Wilton, "Numerical Evaluation of Singular and Near-Singular Potential Integrals," IEEE Transactions on Antennas and Propagation, vol. 53, pp. 31803190, Oct. 2005.
[8] M. Khayat, D. Wilton, and P. Fink, "An improved transformation and optimized sampling scheme for the numerical evaluation of singular and near-singular potentials," Antennas and Wireless Propagation Letters, IEEE, vol. 7, pp. $377-380,2008$.

[9] D. Taylor, "Accurate and efficient numerical integration of weakly singular integrals in galerkin efie solutions," Antennas and Propagation, IEEE Transactions on, vol. 51, no. 7, pp. 1630 - 1637, july 2003.

[10] A. G. Polimeridis, F. Vipiana, J. R. Mosig, and D. R. Wilton, "DIRECTFN: Fully numerical algorithms for high precision computation of singular integrals in Galerkin SIE methods," IEEE Transactions on Antennas and Propagation, 2013.

[11] P. Arcioni, M. Bressan, and L. Perregrini, "On the evaluation of the double surface integrals arising in the application of the boundary integral method to 3-d problems," Microwave Theory and Techniques, IEEE Transactions on, vol. 45, no. 3, pp. $436-439$, mar 1997.

[12] I. Bogaert and D. De Zutter, "High precision evaluation of the selfpatch integral for linear basis functions on flat triangles," IEEE Transactions on Antennas and Propagation, vol. 58, no. 5, pp. 1813-1816, May 2010.

[13] M. Taskinen, "On the fully analytical integration of singular double integrals arising from the integral equation methods," in Computational Electromagnetics International Workshop (CEM), 2011, aug. 2011, pp. $13-18$.

[14] I. Bogaert, "Analytical Computation of Impedance Integrals with Power-Law Green's Functions," in Proceedings of the International Conference on Electromagnetics in Advanced Applications, Cape Town, South Africa, 2-7 September 2012.

[15] A. Peterson, S. Ray, and R. Mittra, Computational methods for electromagnetics. New York: IEEE, 1998.

[16] I. Bogaert, F. Andriulli, and K. Cools, "LowFrequency Stability of the Razor Blade Tested MFIE," in Proceedings of the International Conference on Electromagnetics in Advanced Applications, Turin, Italy, 9-13 September 2013. 